\title{
Clinical Effects of the COVID-19 Pandemic Among the Uninfected Pregnant Women - 6 PLADs, China, 2019-2020
}

\author{
Bin Wang ${ }^{1,2,2}$; Hang An ${ }^{1,2,2}$; Huanqing $\mathrm{Hu}^{3}$; Wei Zhao ${ }^{3}$; Bahabaike Jiangtulu ${ }^{1,2}$; Shuo Wang; ${ }^{4}$ Jiamei Wang ${ }^{4}$; \\ Junxi Chen ${ }^{1,2}$; Manman Long ${ }^{1,2}$; Zewu Li $^{1,2}$; Yu Jin ${ }^{1,2}$; Yuhuan Li $^{1,2}$; Huiting Chen ${ }^{1,2}$; Tao Xue ${ }^{1,2}$; Xiqing Li ${ }^{5}$; \\ Kexin $\mathrm{Li}^{6}$; Wei $\mathrm{Du}^{7}$; Suhong $\mathrm{Gao}^{4}$; Jiangli $\mathrm{Di}^{3, * *}$; Xiaohong $\mathrm{Liu}^{4, * *}$; Rongwei $\mathrm{Ye}^{1, *}$; Zhiwen $\mathrm{Li}^{1,2, *}$
}

\section{Summary \\ What is already known about this topic? \\ The coronavirus disease 2019 (COVID-19) pandemic potentially affected prenatal care quality and maternal and fetal outcomes globally. \\ What is added by this report? \\ During COVID-19 pandemic period, the rates of caesarean sections (CS) and preterm birth for uninfected pregnant women increased slightly in areas that were relatively severely impacted by the pandemic in China. The overall number of prenatal examinations did not dramatically decrease, while the eligible examinations significantly decreased in Hubei Province. \\ What are the implications for public health practice? \\ Routine prenatal examinations had been well maintained during the pandemic period in China. In the future, in-time prenatal examinations should be provided to improve the quality of screening and management of high-risk pregnancy under pandemic- affected circumstances. Psychological counseling and transfer treatment channels should be strengthened for pregnant women during lockdown period.}

The impact of the coronavirus disease 2019 (COVID-19) pandemic on pregnant women have been of considerable concern. Uninfected pregnant women may be affected by generalized social panic, reduced number of prenatal examinations $(\mathrm{NPE})^{*}$, and delayed medical treatment. During the COVID-19 pandemic in China, most pregnant women stayed at home unless an urgent prenatal examination or treatment was required. It was reported that
COVID-19 pandemic resulted in approximately $1 / 3$ of pregnant women having inadequate antenatal visits and $44.7 \%$ of pregnancies with complications due to their fear of contracting infections (1). This study examined the impact of the COVID-19 pandemic on the clinical characteristics of uninfected pregnant women and their neonates by using two ongoing programs conducted by the Maternal and Newborn Health Monitoring Program (MNHMP) and a birth cohort of the Haidian Maternal and Child Care Hospital (HMCCH) in Beijing, China. The results suggested that the rates of caesarean section (CS) and preterm birth $^{\dagger}$ increased slightly in areas that were more affected by the pandemic. NPE were not significantly interrupted, and most maternal and neonatal clinical characteristics were within the normal ranges. The rate of the number of prenatal eligible examinations exceeding 5 times (PEE5) with blood pressure and blood routine examinations during the regulated prenatal care ${ }^{\S}$ according to the National Standards for Basic Public Health Services in China was significantly reduced in Hubei. We concluded that the frequency of routine prenatal care had been well maintained in China during the pandemic period in 2020. However, special in-time prenatal examination should be provided to improve the quality of care for pregnant women, and screening and management of high-risk pregnancies should be strengthened when pandemic-like circumstances occur in the future.

This prospective cohort study was based on two ongoing multicenter programs including: 1) the MNHMP established in 2013 by the National Center for Women and Children's Health (NCWCH) of China CDC, of which the detailed information had been described previously (2); and 2) a birth cohort in

\footnotetext{
* The times of examinations which women took from their first antenatal care examination to childbirth.

${ }^{\dagger}$ Live infants born before 37 gestational weeks.

$\S$ The regulated prenatal care in standards for the management and service of maternal health is issued in the National Standards for Basic Public Health Services (Third Edition) by National Health Commission in China. There are routine prenatal cares for five times at $<13^{\text {rd }}, 16^{\text {th }}-20^{\text {th }}, 21^{s^{s}}-24^{\text {th }}$, $28^{\text {th }}-36^{\text {th }}$, and $37^{\text {st }}-40^{\text {th }}$. The details can be found on the website: http://www.nhc.gov.cn/.
} 
$\mathrm{HMCCH}$ in Beijing in China. For all areas, pregnant women were recruited with delivery or termination from January 1, 2019 to May 31, 2019 (pre-pandemic period: P-2019) and from January 1, 2020 to May 31, 2020 (pandemic period: P-2020). Finally, 32,277 pregnant women were included from 6 Provincial Administration Level Divisions(PLADs), including 6,449 in Hubei Province, 5,878 in Guangdong Province, 3,641 in Hebei Province, 6,328 in Shandong Province, 2,920 in Yunnan Province, and 7,061 in Beijing Municipality. The main outcomes, including the NPE, PEE5, preterm birth, stillbirth", large for gestational age (LGA) ${ }^{* *}$, small for gestational age $(\mathrm{SGA})^{\dagger \dagger}$, macrosomia ${ }^{\S}$, and low birth weight $(\mathrm{LBW})^{\text {१ा }}$ status were recorded or calculated. Means \pm standard deviations (SDs) or medians (25\%-75\% percentile ranges) were used to describe distributions. SGA and LGA status were assessed from gestational week 24-42. COVID-19 pandemic status served as a categorical variable. Three regression models were used to estimate the effects of the pandemic depending on the data distribution, i.e., Poisson, linear, and log-binomial regressions. The confounders included body mass index (BMI, categorical), age (categorical), parity, gravidity, ethnicity, and educational level. The study protocol was approved by the institutional review board of Peking University.

The demographic characteristics of all women were listed in Table 1. The mean ages differed between the two periods (P-2019 vs. P-2020) for Hubei (29.1 vs. 28.7), Guangdong (28.4 vs. 27.9), Beijing (31.6 vs. 31.4), and Hebei (29.8 vs. 29.4). However, the differences were negligible (maximum $1.7 \%$ in Guangdong). Parity distribution varied by location but did not differ between the two periods. The average BMI $\left(\mathrm{kg} / \mathrm{m}^{2}\right)$ ranged from 22.2 (Hubei) to 23.2 (Hebei). For Hebei alone, the average BMI increased slightly from 23.1 (P-2019) to 23.5 (P-2020). Educational levels differed greatly; although significant differences between the two periods were evident in Hubei and Guangdong, the overall pattern remained similar.

The maternal and neonatal clinical characteristics were listed in Table 2. The NPE varied by locations. The average NPE in Hubei and Beijing decreased slightly from P-2019 to P-2020 but increased in Guangdong and Hebei. Comparatively speaking, the ratio of PEE5 significantly decreased from $5.4 \%$ (P2019) to $2.6 \%$ (P2020) in Hubei. No betweenperiod changes in maternal death, stillbirth, neonatal gender, or normal weight or LBW status were evident. The maternal death and stillbirth rates rarely occurred. Guangdong (28\%) and Yunnan (27\%) exhibited lower $\mathrm{CS}$ rates than the other regions. The CS rates increased significantly between the two periods for Hubei and Guangdong only. Most Apgar scores ranged from 9 to 10 except for Hubei monitored at $1 \mathrm{~min}$, but the differences were negligible. The preterm birth rate increased between the two periods for Hubei and Guangdong only. The macrosomia rate in Beijing only increased by about $2 \%$. The SGA rates decreased for Hubei and Guangdong only. The LGA rate in Hubei only increased from $8 \%$ to $10 \%$.

The adjusted regression parameters of the clinical manifestations of the two periods are listed in Table 3, which were consistent with those without adjustment for confounders. For Hubei, the NPE, delivery gestational weeks (DGW) ${ }^{* * *}$, and SGA were negatively associated with the pandemic, and the CS and preterm birth rates were positively associated. Guangdong was similar, but the NPE change lacked significance. The PEE5 in Hubei were negatively associated with the pandemic, while not for others. The effect of the pandemic on preterm birth in Hubei and Guangdong did not change substantially with additional adjustment for CS. In contrast, we only found the positive associations for the macrosomia risk in Beijing and the NPE in Hebei, and a negative association for the DGW in Yunnan.

\section{DISCUSSION}

This study explored the effects of the pandemic on pregnancy-related clinical manifestations in uninfected women. Overall, the effects were minor. However, the significantly increased rates of CS and preterm birth in

\footnotetext{
"Late fetal deaths weighing more than $1,000 \mathrm{~g}$ or occurring after 28 weeks gestation.

** Live infants born at gestational weeks $24-42$ without major birth defects and with birthweight above the $90^{\text {th }}$ percentile for gestational age of the reference population mean for birthweight and gestational age.

${ }^{\dagger}$ Live infants born at gestational weeks $24-42$ without major birth defects and with birthweight below the $10^{\text {th }}$ percentile for gestational age of the reference population mean for birthweight and gestational age.

$\$$ Live infants with weight at birth not less than $4,000 \mathrm{~g}$.

"II Live infants with weight at birth less than 2,500 g.

${ }^{* * *}$ Duration from the last menstrual period reported by the women and the time of delivery.
} 
Hubei and Guangdong with relatively high COVID19 prevalence level requires attention, as the increased risk of macrosomia in Beijing. Thus, our hypothesis is only partly supported.

Many reports on the pregnancy complications and neonatal birth outcomes of infected women have appeared; the preterm birth and CS rates increased significantly in countries with high COVID-19 burdens (3-4). However, the effects on uninfected pregnant women have been rarely reported. In the United Kingdom, it has been proposed that the stillbirth increase was caused by a reluctance to visit hospitals for fear of contracting COVID-19 (5). The medical facility experienced a major decline in all aspects of routine obstetric activities during pandemic period (6). In China, strict prevention and control policies ensured social distancing. Even in areas with low infection rates, all nonessential examinations were to be postponed or reduced.

In our study, the NPE decreased only in Hubei by $5 \%$. In the other five areas, the NPE did not decrease and even increased in Hebei. The pandemic did not increase the stillbirth or LBW rates. Beijing (only) exhibited a slightly increased rate of macrosomia. Similar results were found in Wuhan City in Hubei Province suggesting that most of the clinical manifestations did not increase except for the weight of newborns being significantly heavier during the pandemic period (7). All health services worked well, despite the fact that the pandemic consumed a great deal of medical resources. Since the outbreak, medical resources have been rationally allocated using a Joint Prevention and Control Mechanism prepared by the State Council of China to protect pregnant women (8).

TABLE 1. Demographic characteristics of the participants in the 2 periods of 2019 and 2020 in 6 provincial-level administrative divisions (PLADs) of China.

\begin{tabular}{|c|c|c|c|c|c|c|}
\hline $\begin{array}{l}\text { Demographic } \\
\text { characteristic }\end{array}$ & \multicolumn{2}{|c|}{ Hubei } & \multicolumn{2}{|c|}{ Guangdong } & \multicolumn{2}{|c|}{ Beijing } \\
\hline County or district & \multicolumn{2}{|c|}{ Huangmei, Luotian } & \multicolumn{2}{|c|}{ Longchuan, Zijin } & \multicolumn{2}{|c|}{ Haidian } \\
\hline Year & P-2019 & P-2020 & P-2019 & P-2020 & P-2019 & P-2020 \\
\hline No. of subjects & 3,300 & 3,149 & 3,328 & 2,550 & 3,902 & 3,159 \\
\hline \multirow[t]{2}{*}{ Age (years) } & $28.7 \pm 4.2^{* *}$ & $29.1 \pm 4.3^{\S . \S \S}$ & $27.9 \pm 5.0$ & $28.4 \pm 5.0^{\S, \S \S}$ & $31.4 \pm 4.0$ & $31.6 \pm 4.0^{*}, \S \S$ \\
\hline & $28.0(26.0,31.0)^{\dagger \dagger}$ & $29.0(27.0,32.0)^{\S . ~ П \pi ा ~}$ & $\pi 27.0(24.0,31.0)$ & $28.0(25.0,31.0)^{\S, \text { ๆा }}$ & $31.0(29.0,34.0)$ & $31.0(29.0,34.0)^{\dagger, ~ \text { กा }}$ \\
\hline Missing & $0(0)$ & $2(0)$ & $0(0)$ & $2(0)$ & $0(0)$ & $0(0)$ \\
\hline \multicolumn{7}{|l|}{ Gravidity } \\
\hline 1 time & $1,276(39)$ & $1,220(39)$ & $884(27)$ & $698(27)$ & $1,785(46)$ & $1,422(45)$ \\
\hline$\geq 2$ times & $2,024(61)$ & $1,929(61)$ & $2,444(73)$ & $1,852(73)$ & $2,117(54)$ & $1,737(55)$ \\
\hline Missing & $0(0)$ & $0(0)$ & $0(0)$ & $0(0)$ & $0(0)$ & $0(0)$ \\
\hline \multicolumn{7}{|l|}{ Parity } \\
\hline Nulliparous & $-{ }^{t+\dagger}$ & - & $1,255(38)$ & $960(38)$ & $2,517(65)$ & $2,058(65)$ \\
\hline Multiparous & - & - & $2,073(62)$ & $1,590(62)$ & $1,385(35)$ & $1,101(35)$ \\
\hline Missing & - & - & $0(0)$ & $0(0)$ & $0(0)$ & $0(0)$ \\
\hline \multirow[t]{2}{*}{ BMI $\left(\mathrm{kg} / \mathrm{m}^{2}\right)$} & $22.2 \pm 3.1^{* *}$ & $22.3 \pm 3.2$ & $22.0 \pm 3.3$ & $22.1 \pm 3.5$ & - & - \\
\hline & $21.7(20.1,23.8)^{\dagger \dagger}$ & $21.9(20,24)$ & $21.6(19.6,24.1)$ & $21.7(19.5,24.1)$ & - & - \\
\hline Missing & $116(4)$ & $167(5)$ & $84(3)$ & $87(3)$ & - & - \\
\hline \multicolumn{7}{|l|}{ Education } \\
\hline Primary or lower & $26(1)$ & $15(0)^{\S, * \star}$ & $58(2)$ & $53(2)^{\dagger,{ }^{* \star \star}}$ & - & - \\
\hline Junior high & $558(17)$ & $424(14)$ & $1,865(56)$ & $1,351(53)$ & - & - \\
\hline Senior high & $2,295(72)$ & $2,377(78)$ & $760(23)$ & $566(22)$ & - & - \\
\hline College or higher & $316(10)$ & $242(8)$ & $645(19)$ & $580(23)$ & - & - \\
\hline Missing & $105(3)$ & $91(3)$ & $0(0)$ & $0(0)$ & - & - \\
\hline \multicolumn{7}{|l|}{ Ethnicity } \\
\hline Han & $3,248(100)$ & $3,086(100)$ & $3,233(98)$ & $2,483(98)$ & - & - \\
\hline Others & $10(0)$ & $12(0)$ & $51(2)$ & $47(2)$ & - & - \\
\hline Missing & $42(1)$ & $51(2)$ & $44(1)$ & $20(1)$ & - & - \\
\hline
\end{tabular}


TABLE 1. (Continued)

\begin{tabular}{|c|c|c|c|c|c|c|}
\hline $\begin{array}{l}\text { Demographic } \\
\text { characteristic }\end{array}$ & \multicolumn{2}{|c|}{ Shandong } & \multicolumn{2}{|c|}{ Hebei } & \multicolumn{2}{|c|}{ Yunnan } \\
\hline County or district & \multicolumn{2}{|c|}{ Shizhong, Huaiyin } & \multicolumn{2}{|c|}{ Zhengding, Xingtai } & \multicolumn{2}{|c|}{ Huaning, Tonghai } \\
\hline Year & P-2019 & P-2020 & P-2019 & P-2020 & P-2019 & P-2020 \\
\hline No. of subjects & 3,879 & 2,449 & 2,280 & 1,361 & 1,952 & 968 \\
\hline \multirow[t]{2}{*}{ Age (years) } & $31.3 \pm 4.3$ & $31.3 \pm 4.2$ & $29.4 \pm 3.9$ & $29.8 \pm 3.9^{\dagger, \S \S}$ & $27.9 \pm 4.8$ & $28.0 \pm 4.6$ \\
\hline & $31.0(29.0,34.0)$ & $31.0(29.0,34.0)$ & $29.0(27.0,32.0)$ & $30.0(27.0,32.0)^{\dagger, \pi \pi}$ & $28.0(24.0,31.0)$ & $28.0(25.0,31.0)$ \\
\hline Missing & $0(0)$ & $0(0)$ & $0(0)$ & $1(0)$ & $0(0)$ & $0(0)$ \\
\hline \multicolumn{7}{|l|}{ Gravidity } \\
\hline 1 time & $1,353(35)$ & $832(34)$ & $562(25)$ & $317(23)$ & $537(28)$ & $227(23)^{*}, \ldots * t$ \\
\hline$\geq 2$ times & $2,526(65)$ & $1,617(66)$ & $1,718(75)$ & $1,044(77)$ & $1,415(72)$ & $741(77)$ \\
\hline Missing & $0(0)$ & $0(0)$ & $0(0)$ & $0(0)$ & $0(0)$ & $0(0)$ \\
\hline \multicolumn{7}{|l|}{ Parity } \\
\hline Nulliparous & $1,968(51)$ & $1,182(48)$ & $764(34)$ & $465(34)$ & $772(40)$ & $390(40)$ \\
\hline Multiparous & $1,911(49)$ & $1,267(52)$ & $1,516(66)$ & $896(66)$ & $1,180(60)$ & $578(60)$ \\
\hline Missing & $0(0)$ & $0(0)$ & $0(0)$ & $0(0)$ & $0(0)$ & $0(0)$ \\
\hline \multirow[t]{2}{*}{$\operatorname{BMI}\left(\mathrm{kg} / \mathrm{m}^{2}\right)$} & $22.9 \pm 3.6$ & $22.8 \pm 3.6$ & $23.1 \pm 4.0$ & $23.5 \pm 4.2$ & $22.1 \pm 3.7$ & $22.1 \pm 3.8$ \\
\hline & $22.3(20.2,24.8)$ & $22.3(20.3,24.6)$ & $22.4(20.2,25.3)$ & $22.8(20.4,26.1)^{\dagger, \text { กाก }}$ & $21.4(19.4,24.1)$ & $21.4(19.2,24.2)$ \\
\hline Missing & $36(1)$ & $9(0)$ & $1(0)$ & $0(0)$ & $0(0)$ & $0(0)$ \\
\hline \multicolumn{7}{|l|}{ Education } \\
\hline Primary or lower & $9(0)$ & $9(0)$ & $19(1)$ & $4(0)$ & $199(10)$ & $86(9)$ \\
\hline Junior high & $237(7)$ & $138(7)$ & $457(25)$ & $277(24)$ & $700(36)$ & $372(39)$ \\
\hline Senior high & $477(14)$ & $240(12)$ & $479(26)$ & $310(27)$ & $564(29)$ & $289(30)$ \\
\hline College or higher & $2,687(79)$ & $1,675(81)$ & $895(48)$ & $551(48)$ & $475(25)$ & $211(22)$ \\
\hline Missing & $469(12)$ & $387(16)$ & $430(19)$ & $219(16)$ & $14(1)$ & $10(1)$ \\
\hline \multicolumn{7}{|l|}{ Ethnicity } \\
\hline Han & $3,740(98)$ & $2,375(98)$ & $1,819(98)$ & $1,124(99)$ & $1,514(78)$ & $744(78)$ \\
\hline Others & $79(2)$ & $54(2)$ & $31(2)$ & $17(1)$ & $419(22)$ & $216(22)$ \\
\hline Missing & $60(2)$ & $20(1)$ & 430 (19) & $220(16)$ & $19(1)$ & $8(1)$ \\
\hline
\end{tabular}

Notes: The COVID-19 pandemic level of the 6 PLADs are: Hubei, level IV; Guangdong, level III; Beijing, level II; Shandong, level II; Hebei, level I; and Yunnan, level I.

Abbreviations: P-2019, January 1-May 31, 2019 as the pre-pandemic period; P-2020, January 1-May 31, 2020 as the pandemic period.

* $P<0.05$;

$+P<0.01$

$\S P<0.001$;

"The COVID-19 pandemic levels were classified according to the provincial overall prevalence rate compared to Hubei Province;;

${ }^{* *}$ Mean value \pm standard deviation;

${ }^{t \dagger}$ Median value (25\%-75\% percentile);

$\S \S$ Compared by $t$ test;

กा Compared by Mann-Whitney U test;

*** Compared by Chi-square test;

${ }^{\dagger \dagger \dagger}$ Data was unavailable.

Overall, the pandemic did not compromise routine prenatal examinations in China.

Notably, the CS rate increased in Hubei and Guangdong during the pandemic period. One possible explanation is that the number of high-risk pregnant women with pregnancy complications may increase due to delayed prenatal examinations and in-time interventions during the pandemic. This viewpoint was supported by the decreased PEE5 in Hubei. In India, delayed health-seeking behaviors during pandemic period resulted in $44.7 \%$ of pregnancies having complications, roughly $1 / 3$ of women having an inadequate number of antenatal visits, and a 2.5-fold rise in admission to the intensive care unit of pregnant women (1). Another possible explanation is that the pandemic increased stress and anxiety. In China, a study conducted in 25 hospitals across China during January 1-February 9, 2020 found that the pandemic increased the risk of depressive and anxiety symptoms (9). Perhaps more women requested CS to ensure a live birth. In Wuhan, maternal requested CS significantly increased during the lockdown period ( 7 ). For the risk 
TABLE 2. Maternal and neonatal clinical characteristics in the two periods of 2019 and 2020 in 6 provincial-level administrative divisions (PLADs) of China.

\begin{tabular}{|c|c|c|c|c|c|c|}
\hline \multirow{2}{*}{ Characteristic } & \multicolumn{2}{|c|}{ Hubei } & \multicolumn{2}{|c|}{ Guangdong } & \multicolumn{2}{|c|}{ Beijing } \\
\hline & P-2019 & P-2020 & P-2019 & P-2020 & P-2019 & P-2020 \\
\hline $\mathrm{N} 1 \pi$ & 3,300 & 3,149 & 3,328 & 2,550 & 3,902 & 3,159 \\
\hline \multicolumn{7}{|c|}{ No. of prenatal examinations } \\
\hline Mean (SD) & $5.3 \pm 2.5^{\star \star}$ & $5.0 \pm 2.0^{\S, \S \S}$ & $5.2 \pm 2.5$ & $5.4 \pm 2.5^{\S, \S \S}$ & $15.0 \pm 2.8$ & $14.0 \pm 3.1^{\S, \S \S}$ \\
\hline Median (IQR) & $5(4,6.8)^{\dagger \dagger}$ & $5(4,6)^{\S, ~ ๆ \pi ा ~}$ & $5(3,7)$ & $5(3,7)^{\S, ~ ๆ ก ा ~}$ & $15(13,17)$ & $14(13,16)^{\S, ~ ๆ ศ ~}$ \\
\hline Missing & $0(0)$ & $0(0)$ & $0(0)$ & $0(0)$ & $0(0)$ & $0(0)$ \\
\hline \multicolumn{7}{|c|}{ Prenatal eligible examinations ( $>5$ times) } \\
\hline No & $3,123(94.6)$ & $3,067(97.4)^{\S, * \star *}$ & $3,270(98.3)$ & $2,494(97.8)$ & - & - \\
\hline Yes & $177(5.4)$ & $82(2.6)$ & $58(1.7)$ & $56(2.2)$ & - & - \\
\hline Missing & $0(0)$ & $0(0)$ & $0(0)$ & $0(0)$ & - & - \\
\hline \multicolumn{7}{|c|}{ Delivery gestational weeks } \\
\hline Mean $\pm S D$ & $39.0 \pm 1.3^{\star *}$ & $38.9 \pm 1.3^{\S, \S \S}$ & $39.2 \pm 1.4^{* *}$ & $39.0 \pm 1.4^{\S, \S \S}$ & $39.11 \pm 1.3$ & $39.10 \pm 1.4$ \\
\hline Median (IQR) & $39.0(38.0,40.0)^{\dagger \dagger}$ & $39.0(38.0,40.0)^{\S, \pi \pi}$ & $39.3(38.4,40.1)^{\dagger \dagger}$ & $39.1(38.4,40.0)^{\S, ~ ๆ \pi ~}$ & $39.3(38.6,39.9)$ & $39.1(38.6,40.0)$ \\
\hline Missing & $0(0)$ & $0(0)$ & $0(0)$ & $0(0)$ & $0(0)$ & $0(0)$ \\
\hline \multicolumn{7}{|c|}{ Caesarean section (CS) } \\
\hline No & $1,738(53)$ & $1,449(46)^{\S, ~ * \star \star}$ & $2,483(75)$ & $1,761(69)^{\S, * \star}$ & $2,273(58)$ & $1,804(57)$ \\
\hline Yes & $1,562(47)$ & $1,700(54)$ & $845(25)$ & $789(31)$ & $1,629(42)$ & $1,355(43)$ \\
\hline Missing & $0(0)$ & $0(0)$ & $0(0)$ & $0(0)$ & $0(0)$ & $0(0)$ \\
\hline \multicolumn{7}{|l|}{ Maternal death } \\
\hline No & - & - & $3,328(100)$ & $2,550(100)$ & $3,902(100)$ & $3,159(100)$ \\
\hline Yes & - & - & $0(0)$ & $0(0)$ & $0(0)$ & $0(0)$ \\
\hline Missing & - & - & $0(0)$ & $0(0)$ & $0(0)$ & $0(0)$ \\
\hline \multicolumn{7}{|l|}{ Stillbirth (\%) } \\
\hline No & $3,293(100)$ & $3,143(100)$ & $3,326(100)$ & $2,549(100)$ & $3,900(100)$ & $3,155(100)$ \\
\hline Yes & $7(0)$ & $6(0)$ & $2(0)$ & $1(0)$ & $2(0)$ & $4(0)$ \\
\hline Missing & $0(0)$ & $0(0)$ & $0(0)$ & $0(0)$ & $0(0)$ & $0(0)$ \\
\hline $\mathrm{N} 2^{\pi}$ & 3,293 & 3,143 & 3,326 & 2,549 & 3,900 & 3,155 \\
\hline \multicolumn{7}{|l|}{ Neonatal gender } \\
\hline Female & $1,548(47)$ & $1,434(46)$ & $1,561(47)$ & $1,192(47)$ & $1,886(48)$ & $1,547(49)$ \\
\hline Male & $1,745(53)$ & $1,709(54)$ & $1,765(53)$ & $1,356(53)$ & $2,014(52)$ & $1,608(51)$ \\
\hline Unknown & $0(0)$ & $0(0)$ & $0(0)$ & $1(0)$ & $0(0)$ & $0(0)$ \\
\hline Missing & $0(0)$ & $0(0)$ & $0(0)$ & $0(0)$ & $0(0)$ & $0(0)$ \\
\hline \multicolumn{7}{|c|}{ Neonatal weight $(\mathrm{kg})$} \\
\hline Mean $\pm S D$ & $3.32 \pm 0.4$ & $3.33 \pm 0.4$ & $3.16 \pm 0.4$ & $3.17 \pm 0.4$ & $3.33 \pm 0.4$ & $3.32 \pm 0.4$ \\
\hline Median (IQR) & $3.30(3.00,3.60)$ & $3.30(3.00,3.60)$ & $3.10(2.90,3.40)$ & $3.20(2.90,3.50)$ & $3.35(3.08,3.60)$ & 3.32 (3.08 3.60) \\
\hline Missing & $0(0)$ & $0(0)$ & $0(0)$ & $0(0)$ & $0(0)$ & $0(0)$ \\
\hline \multicolumn{7}{|l|}{ Apgar score } \\
\hline $1 \min (S D)$ & $8.7 \pm 0.8$ & $8.9 \pm 0.6^{\S, \S \S}$ & $9.4 \pm 0.6$ & $9.4 \pm 0.6^{\star}, \S \S$ & $10 \pm 0.4$ & $9.9 \pm 0.5$ \\
\hline Missing & $454(14)$ & $586(19)$ & $0(0)$ & $0(0)$ & $2(0)$ & $2(0)$ \\
\hline $5 \min (S D)$ & $9.5 \pm 0.6$ & $9.4 \pm 0.6^{\S, \S \S}$ & $10 \pm 0.2$ & $10 \pm 0.2$ & $10 \pm 0.3$ & $10 \pm 0.3$ \\
\hline Missing & $447(14)$ & $583(19)$ & $0(0)$ & $0(0)$ & $2(0)$ & $2(0)$ \\
\hline $10 \min (\mathrm{SD})$ & $9.5 \pm 0.5$ & $9.9 \pm 0.4^{\S, \S \S}$ & $10 \pm 0.1$ & $10 \pm 0.1$ & $10 \pm 0.4$ & $10 \pm 0.4$ \\
\hline Missing & $992(30)$ & $672(21)$ & $0(0)$ & $0(0)$ & $13(0)$ & $15(1)$ \\
\hline Preterm birth & & & & & & \\
\hline No & $3,212(98)$ & $3,036(97)^{*, * * \star}$ & $3,180(96)$ & $2,399(94)^{\dagger, * \star \star}$ & $3,729(96)$ & $3,011(95)$ \\
\hline Yes & $81(2)$ & $107(3)$ & $146(4)$ & $150(6)$ & $171(4)$ & $144(5)$ \\
\hline Missing & $0(0)$ & $0(0)$ & $0(0)$ & $0(0)$ & $0(0)$ & $0(0)$ \\
\hline Low birth weight & & & & & & \\
\hline No & $3,235(98)$ & $3,083(98)$ & $3,194(96)$ & $2,434(96)$ & $3,788(97)$ & 3,049 (97) \\
\hline Yes & $58(2)$ & $60(2)$ & $132(4)$ & $115(4)$ & $112(3)$ & $106(3)$ \\
\hline Missing & $0(0)$ & $0(0)$ & $0(0)$ & $0(0)$ & $0(0)$ & $0(0)$ \\
\hline Macrosomia & & & & & & \\
\hline No & $3,093(94)$ & $2,923(93)$ & $3,239(97)$ & $2,467(97)$ & $3,728(96)$ & $2,977(94)^{*}, * \star \star$ \\
\hline Yes & $200(6)$ & $220(7)$ & $87(3)$ & $82(3)$ & $172(4)$ & $178(6)$ \\
\hline Missing & $0(0)$ & $0(0)$ & $0(0)$ & $0(0)$ & $0(0)$ & $0(0)$ \\
\hline$N 3^{\pi}$ & 3,269 & 3,118 & 3,322 & 2,529 & 3,857 & 3,105 \\
\hline Small for gestation & al age & & & & & \\
\hline No & $3,029(93)$ & $2,929(94)^{*, * \star * \star}$ & $2,898(87)$ & $2,310(91)^{\S, * \star \star}$ & $3,688(96)$ & $2,967(96)$ \\
\hline Yes & $240(7)$ & $189(6)$ & $424(13)$ & $219(9)$ & $169(4)$ & $138(4)$ \\
\hline Missing & $0(0)$ & $0(0)$ & $0(0)$ & $0(0)$ & $0(0)$ & $0(0)$ \\
\hline Large for gestation & al age & & & & & \\
\hline No & $3,007(92)$ & $2,817(90)^{\star \star * \star \star \star}$ & $3,179(96)$ & $2,404(95)$ & $3,531(919)$ & $2,811(91)$ \\
\hline Yes & $262(8)$ & $301(10)$ & $143(4)$ & $125(5)$ & $326(9)$ & $294(9)$ \\
\hline Missing & $0(0)$ & $0(0)$ & $0(0)$ & $0(0)$ & $0(0)$ & $0(0)$ \\
\hline
\end{tabular}


TABLE 2. (Continued)

\begin{tabular}{|c|c|c|c|c|c|c|}
\hline \multirow{2}{*}{ Characteristic } & \multicolumn{2}{|c|}{ Shandong } & \multicolumn{2}{|c|}{ Hebei } & \multicolumn{2}{|c|}{ Yunnan } \\
\hline & P-2019 & P-2020 & P-2019 & P-2020 & P-2019 & P-2020 \\
\hline $\begin{array}{l}\mathrm{N} 1^{\top} \\
\text { Number of prenatal }\end{array}$ & $\begin{array}{l}3,879 \\
\text { xaminations }\end{array}$ & 2,449 & 2,280 & 1,361 & 1,952 & 968 \\
\hline Mean $\pm S D$ & - & - & $5.5 \pm 3.0^{* *}$ & $6.3 \pm 2.8^{\S, \S \S}$ & $11.5 \pm 2.6$ & $11.6 \pm 2.4$ \\
\hline $\begin{array}{l}\text { Median (IQR) } \\
\text { Missing }\end{array}$ & $\begin{array}{l}- \\
-\end{array}$ & $\begin{array}{l}- \\
-\end{array}$ & $\begin{array}{l}6(2,8)^{\dagger \dagger} \\
0(0)\end{array}$ & $\begin{array}{l}7(4,8)^{\S, ~ \pi T \pi} \\
0(0)\end{array}$ & $\begin{array}{l}11(10,13) \\
0(0)\end{array}$ & $\begin{array}{l}12(10,13) \\
0(0)\end{array}$ \\
\hline \multicolumn{7}{|c|}{ Prenatal eligible examinations (>5 times) } \\
\hline $\begin{array}{l}\text { No } \\
\text { Yes } \\
\text { Missing }\end{array}$ & $\begin{array}{l}- \\
- \\
-\end{array}$ & $\begin{array}{l}- \\
- \\
-\end{array}$ & $\begin{array}{l}1,606(70.4) \\
674(29.6) \\
0(0)\end{array}$ & $\begin{array}{l}960(70.5) \\
401(29.5) \\
0(0)\end{array}$ & $\begin{array}{l}779(39.9) \\
1,173(60.1) \\
0(0)\end{array}$ & $\begin{array}{l}249(25.7)^{\S,{ }^{* \star \star}} \\
719(74.3) \\
0(0)\end{array}$ \\
\hline \multicolumn{7}{|c|}{ Delivery gestational weeks } \\
\hline $\begin{array}{l}\text { Mean } \pm S D \\
\text { Median (IQR) } \\
\text { Missing }\end{array}$ & $\begin{array}{l}39.2 \pm 1.3 \\
39.3(38.6,40.1) \\
0(0)\end{array}$ & $\begin{array}{l}39.2 \pm 1.3 \\
39.3(38.6,40.1) \\
0(0)\end{array}$ & $\begin{array}{l}39.1 \pm 1.4 \\
39.3(38.6,40.0) \\
0(0)\end{array}$ & $\begin{array}{l}39.2 \pm 1.5 \\
39.3(38.6,40.0) \\
0(0)\end{array}$ & $\begin{array}{l}39.0 \pm 1.4 \\
39.0(38.3,40.0) \\
0(0)\end{array}$ & $\begin{array}{l}38.9 \pm 1.4 \\
39.0(38.0,40.0) \\
0(0)\end{array}$ \\
\hline \multicolumn{7}{|l|}{ Caesarean section } \\
\hline $\begin{array}{l}\text { No } \\
\text { Yes } \\
\text { Missing }\end{array}$ & $\begin{array}{l}1,947(50) \\
1,932(50) \\
0(0)\end{array}$ & $\begin{array}{l}1,256(51) \\
1,193(49) \\
0(0)\end{array}$ & $\begin{array}{l}1,381(60) \\
899(40) \\
0(0)\end{array}$ & $\begin{array}{l}808(60) \\
552(40) \\
1(0)\end{array}$ & $\begin{array}{l}1,439(74) \\
512(26) \\
1(0)\end{array}$ & $\begin{array}{l}693(72) \\
274(28) \\
1(0)\end{array}$ \\
\hline \multicolumn{7}{|l|}{ Maternal death } \\
\hline $\begin{array}{l}\text { No } \\
\text { Yes } \\
\text { Missing }\end{array}$ & $\begin{array}{l}3,879(100) \\
0(0) \\
0(0)\end{array}$ & $\begin{array}{l}2,449(100) \\
0(0) \\
0(0)\end{array}$ & $\begin{array}{l}2,280(100) \\
0(0) \\
0(0)\end{array}$ & $\begin{array}{l}1,359(100) \\
2(0) \\
0(0)\end{array}$ & $\begin{array}{l}1,748(100) \\
1(0) \\
203(10)\end{array}$ & $\begin{array}{l}909(100) \\
1(0) \\
58(6)\end{array}$ \\
\hline \multicolumn{7}{|l|}{ Stillbirth $(\%)$} \\
\hline $\begin{array}{l}\text { No } \\
\text { Yes } \\
\text { Missing }\end{array}$ & $\begin{array}{l}3,879(100) \\
0(0) \\
0(0)\end{array}$ & $\begin{array}{l}2,449(100) \\
0(0) \\
0(0)\end{array}$ & $\begin{array}{l}2,277(100) \\
3(0) \\
0(0)\end{array}$ & $\begin{array}{l}1,360(100) \\
1(0) \\
0(0)\end{array}$ & $\begin{array}{l}1,946(100) \\
6(0) \\
0(0)\end{array}$ & $\begin{array}{l}965(100) \\
3(0) \\
0(0)\end{array}$ \\
\hline $\begin{array}{l}\mathrm{N} 2{ }^{\pi} \\
\text { Neonatal gender }\end{array}$ & 3879 & 2449 & 2277 & 1360 & 1946 & 965 \\
\hline $\begin{array}{l}\text { Female } \\
\text { Male } \\
\text { Unknown } \\
\text { Missing }\end{array}$ & $\begin{array}{l}1,889(49) \\
1,988(51) \\
2(0) \\
0(0)\end{array}$ & $\begin{array}{l}1,166(48) \\
1,282(52) \\
1(0) \\
0(0)\end{array}$ & $\begin{array}{l}1,096(48) \\
1,181(52) \\
0(0) \\
0(0)\end{array}$ & $\begin{array}{l}658(48) \\
702(52) \\
0(0) \\
0(0)\end{array}$ & $\begin{array}{l}930(48) \\
1,016(52) \\
0(0) \\
0(0)\end{array}$ & $\begin{array}{l}450(47) \\
515(53) \\
0(0) \\
0(0)\end{array}$ \\
\hline \multicolumn{7}{|c|}{ Neonatal weight $(\mathrm{kg})$} \\
\hline $\begin{array}{l}\text { Mean } \pm S D \\
\text { Median (IQR) } \\
\text { Missing }\end{array}$ & $\begin{array}{l}3.40 \pm 0.4 \\
3.40(3.10,3.70) \\
0(0)\end{array}$ & $\begin{array}{l}3.40 \pm 0.4 \\
3.40(3.10,3.70) \\
1(0)\end{array}$ & $\begin{array}{l}3.33 \pm 0.5 \\
3.30(3.00,3.60) \\
8(0)\end{array}$ & $\begin{array}{l}3.32 \pm 0.5 \\
3.30(3.00,3.60) \\
12(1)\end{array}$ & $\begin{array}{l}3.23 \pm 0.5 \\
3.20(3.00,3.50) \\
0(0)\end{array}$ & $\begin{array}{l}3.22 \pm 0.4 \\
3.20(3.00,3.50) \\
0(0)\end{array}$ \\
\hline \multicolumn{7}{|l|}{ Apgar score } \\
\hline $\begin{array}{l}1 \text { min } \\
\text { Missing }\end{array}$ & $\begin{array}{l}- \\
-\end{array}$ & $\begin{array}{l}- \\
-\end{array}$ & $\begin{array}{l}9.9 \pm 0.4 \\
110(5)\end{array}$ & $\begin{array}{l}9.9 \pm 0.4 \\
17(1)\end{array}$ & $\begin{array}{l}9.3 \pm 1.4 \\
1(0)\end{array}$ & $\begin{array}{l}9.5 \pm 1^{\dagger, \S \S} \\
0(0)\end{array}$ \\
\hline $\begin{array}{l}5 \text { min } \\
\text { Missing }\end{array}$ & $\begin{array}{l}- \\
-\end{array}$ & $\begin{array}{l}- \\
-\end{array}$ & $\begin{array}{l}10 \pm 0.3 \\
110(5)\end{array}$ & $\begin{array}{l}10 \pm 0.3 \\
17(1)\end{array}$ & $\begin{array}{l}9.8 \pm 1.2 \\
1(0)\end{array}$ & $\begin{array}{l}9.9 \pm 0.7^{\dagger, \S \S} \\
0(0)\end{array}$ \\
\hline $\begin{array}{l}10 \text { min } \\
\text { Missing }\end{array}$ & - & - & $\begin{array}{l}10 \pm 0.3 \\
110(5)\end{array}$ & $\begin{array}{l}10 \pm 0.1 \\
17(1)\end{array}$ & $\begin{array}{l}9.8 \pm 1.2 \\
1(0)\end{array}$ & $\begin{array}{l}9.9 \pm 0.8^{*}, \S \S \\
0(0)\end{array}$ \\
\hline \multicolumn{7}{|l|}{ Preterm birth } \\
\hline No & $3,710(96)$ & $2,359(96)$ & $2,174(95)$ & $1,293(95)$ & $1,845(95)$ & $929(96)$ \\
\hline Yes & $169(4)$ & $90(4)$ & $103(5)$ & $67(5)$ & $101(5)$ & $36(4)$ \\
\hline Missing & $0(0)$ & $0(0)$ & $0(0)$ & $0(0)$ & $0(0)$ & $0(0)$ \\
\hline \multicolumn{7}{|l|}{ Low birth weight } \\
\hline No & $3,790(98)$ & $2,397(98)$ & $2,199(97)$ & $1,312(97)$ & $1,863(96)$ & $934(97)$ \\
\hline Yes & $89(2)$ & $51(2)$ & $70(3)$ & $36(3)$ & $83(4)$ & $31(3)$ \\
\hline Missing & $0(0)$ & $1(0)$ & $8(0)$ & $12(1)$ & $0(0)$ & $0(0)$ \\
\hline \multicolumn{7}{|l|}{ Macrosomia } \\
\hline No & $3,540(91)$ & $2,252(92)$ & $2,117(93)$ & $1,266(94)$ & $1,864(96)$ & $932(97)$ \\
\hline Yes & $339(9)$ & $196(8)$ & $152(7)$ & $82(6)$ & $82(4)$ & $33(3)$ \\
\hline Missing & $0(0)$ & $1(0)$ & $8(0)$ & $12(1)$ & $0(0)$ & $0(0)$ \\
\hline N3 ${ }^{\pi}$ & 3877 & 2448 & 1844 & 1138 & 1900 & 953 \\
\hline \multicolumn{7}{|c|}{ Small for gestational age } \\
\hline No & $3,720(96)$ & $2,351(96)$ & $1,759(95)$ & $1,078(95)$ & $1,717(90)$ & $875(92)$ \\
\hline Yes & $155(4)$ & $95(4)$ & $85(5)$ & $60(5)$ & $183(10)$ & $78(8)$ \\
\hline Missing & $2(0)$ & $2(0)$ & $0(0)$ & $0(0)$ & $0(0)$ & $0(0)$ \\
\hline \multicolumn{7}{|c|}{ Large for gestational age } \\
\hline $\begin{array}{l}\text { No } \\
\text { Yes }\end{array}$ & $\begin{array}{l}3,358(87) \\
517(13)\end{array}$ & $\begin{array}{l}2,137(87) \\
309(13)\end{array}$ & $\begin{array}{l}1,648(89) \\
196(11)\end{array}$ & $\begin{array}{l}1,038(91) \\
100(9)\end{array}$ & $\begin{array}{l}1,783(94) \\
117(6)\end{array}$ & $\begin{array}{l}900(94) \\
53(6)\end{array}$ \\
\hline Missing & $2(0)$ & $2(0)$ & $0(0)$ & $0(0)$ & $0(0)$ & $0(0)$ \\
\hline
\end{tabular}

Abbreviations: P-2019, January 1-May 31, 2019 as the pre-pandemic period; P-2020, January 1-May 31, 2020 as the pandemic period.

${ }^{*} P<0.05$;

$\dagger P<0.01$

$\S P<0.001$;

I N1: number of the total subjects, N2: those N1 without delivering stillbirths; and N3: those N2 delivering fetuses at gestational weeks 24-42 and without major birth defects;

${ }^{* *}$ Mean value \pm standard deviation (SD);

${ }^{\dagger \dagger}$ Median value (25\%-75\% percentile);

$\S \S$ Compared by $t$ test;

กा Compared by Mann-Whitney U test;

${ }^{* \star \star}$ Compared by Chi-square test;

${ }^{\mathrm{tt \dagger}}$ Data are unavailable. 
TABLE 3. Adjusted relative risk associated with the occurrence of the COVID-19 between 2019 and 2020 in 6 provinciallevel administrative divisions (PLADs) of China.

\begin{tabular}{|c|c|c|c|}
\hline Characteristics & Hubei & Guangdong & Beijing \\
\hline NPE $E^{\pi} \S \S$ & $0.95(0.92,0.99)^{\dagger}$ & $1.03(1.00,1.07)$ & $0.99(0.96,1.02)$ \\
\hline PEE5 $5^{* *}, \S \S$ & $0.49(0.37,0.64)^{\S}$ & $1.22(0.84,1.76)$ & - \\
\hline $\mathrm{DGW}^{\dagger+}, \S \S$ & $-0.15(-0.22,-0.08)^{\S}$ & $-0.16(-0.23,-0.08)^{\S}$ & $0(-0.02,0.02)$ \\
\hline $\mathrm{CS}^{* *}, \S \S$ & $1.11(1.06,1.17)^{\S}$ & $1.17(1.08,1.27)^{\S}$ & $1.02(0.97,1.08)$ \\
\hline $\mathrm{NW}^{\dagger+}$, $\S$ & $0(-0.02,0.02)$ & $0.01(-0.01,0.03)$ & $-0.01(-0.07,0.05)$ \\
\hline Stillbirth ${ }^{* *}, \S \S$ & $0.88(0.30,2.63)$ & $1.5(0.09,24.47)$ & $2.43(0.44,13.28)$ \\
\hline Preterm birth ${ }^{* *}, \S \S$ & $1.37(1.02,1.84)^{*}$ & $1.34(1.07,1.67)^{*}$ & $1.03(0.83,1.28)$ \\
\hline $\mathrm{LBW}^{* *}, \S \S$ & $1.08(0.75,1.58)$ & $1.18(0.92,1.51)$ & $1.16(0.89,1.50)$ \\
\hline Macrosomia ${ }^{* * *}, \S$ & $1.10(0.91,1.34)$ & $1.18(0.87,1.59)$ & $1.26(1.03,1.55)^{*}$ \\
\hline$S G A^{* *}, \S \S$ & $0.81(0.67,0.98)^{*}$ & $0.71(0.60,0.83)^{\S}$ & $1.01(0.81,1.25)$ \\
\hline $\operatorname{LGA}^{* *}, \S \S$ & $1.17(0.99,1.38)$ & $1.10(0.87,1.39)$ & $1.11(0.96,1.29)$ \\
\hline Characteristics & Shandong & Hebei & Yunnan \\
\hline NPE ${ }^{\pi, ~ \S \S ~}$ & - & $1.08(1.03,1.13)^{\S}$ & $1.0(0.96,1.05)$ \\
\hline PEE5 $5^{\star \star}, \S \S$ & - & $0.96(0.82,1.12)$ & $1.97(1.65,2.34)^{\S}$ \\
\hline $\mathrm{DGW}^{\dagger+}$, $\S$ & $-0.02(-0.09,0.05)$ & $0(-0.11,0.1)$ & $-0.13(-0.23,-0.02)^{*}$ \\
\hline $\mathrm{CS}^{* *}, \S \S$ & $0.99(0.94,1.04)$ & $0.99(0.92,1.07)$ & $1.05(0.93,1.19)$ \\
\hline $\mathrm{NW}^{\dagger+}, \S \S$ & $-0.01(-0.03,0.02)$ & $-0.02(-0.06,0.01)$ & $-0.01(-0.04,0.03)$ \\
\hline Stillbirth ${ }^{* *}, \S \S$ & - & $0.58(0.06,5.55)$ & $0.66(0.13,3.31)$ \\
\hline Preterm birth ${ }^{* *}, \S \S$ & $0.85(0.64,1.11)$ & $1.01(0.72,1.42)$ & $0.73(0.50,1.06)$ \\
\hline $\mathrm{LBW}^{* *}, \S \S$ & $0.98(0.67,1.43)$ & $0.75(0.48,1.18)$ & $0.77(0.51,1.16)$ \\
\hline Macrosomia ${ }^{* *}, \S \S$ & $0.88(0.73,1.06)$ & $0.86(0.65,1.14)$ & $0.83(0.56,1.24)$ \\
\hline$S_{G A}^{* *}, \S \S$ & $0.98(0.74,1.30)$ & $1.13(0.82,1.55)$ & $0.87(0.67,1.12)$ \\
\hline $\operatorname{LGA}^{* *}, \S \S$ & $0.93(0.81,1.08)$ & $0.82(0.65,1.03)$ & $0.87(0.63,1.19)$ \\
\hline
\end{tabular}

Abbreviations: NPE, No. of prenatal examinations; PEE5, Prenatal eligible examinations of $\geq 5$ times; DGW, delivery gestational weeks; CS, Caesarean section; NW, Neonatal weight; LBW, low birth weight; SGA, small for gestational age; LGA, large for gestational age.

${ }^{*} P<0.05$;

${ }^{\dagger} P<0.01$;

$\S P<0.001$;

${ }^{\pi}$ Relative risk calculated by Poisson regression model;

${ }^{* *}$ Relative risk calculated by log-binomial regression model;

${ }^{t \dagger}$ Regression coefficient ( $\beta$ value) calculated by linear regression model;

$\S \S$ Adjusted for BMI (categorical), age (categorical), parity, gravidity, ethnicity, and education. For Hubei Province, parity information is unavailable. For Beijing, the information of BMI, ethnicity, and education is unavailable.

of preterm birth in Hubei and Guangdong, it increased regardless of their higher CS rates. According to clinical practices, women with preterm birth usually had certain pregnancy complications. Though, the routine NPE had been maintained during the pandemic period in China, the prenatal care quality could not be ensured. It is speculated that certain pregnancy complications, drug misuse, or abnormal syndromes may not be treated as usual, which can trigger preterm birth. Therefore, special in-time prenatal examinations should be provided to improve prenatal care quality with certain pregnancy complications when a pandemic occurs in future.
The results of this study were subject to some limitations. First, this study only included 11 countylevel study settings in 6 PLADs to represent a nationwide survey. Second, the asymptomatic infection cases were not excluded by the RNA test. However, we employed a population-based monitoring system by $\mathrm{HMCCH}$ with high-quality data. Also, the prevalence rate of asymptomatic infections in China had been at a very low level to $10^{-6}-10^{-4}(10)$, which does not affect our statistical results for the non-infected pregnant women. In summary, prenatal examinations proceeded as normal and most maternal and neonatal clinical characteristics were within normal ranges. It should be 
noted that the prenatal care quality may be reduced due to limitations in medical resources. Only specific interventions were additionally required to protect pregnant women with pregnancy complications living in regions with a high burden of COVID-19 pandemic.

Psychological counseling and transfer treatment channel should be strengthened for those at high risk during lockdown. For example, online psychological counseling can be used as a good auxiliary method during severe pandemic conditions.

Acknowledgements: Dr. Aiqun Huang, Dr. Xiaoping Pan, and Dr. Jianli Ye of China CDC; local healthcare workers in the 6 PLADs of Yunnan Province (Huaning County and Tonghai County), Hebei Province (Zhengding County and Xingtai County), Shandong Province (Shizhong District and Huaiyin District), Hubei Province (Huangmei County and Luotian County), and Guangdong Province (Longchuan Country and Zijin County), Beijing City (Haidian District); the working group of environmental exposure and human health of the China Cohort Consortium (http://chinacohort.bjmu. edu.cn/).

Conflicts of Interest: All authors declared to have no conflicts of interest.

Ethics Approval: The Peking University ethics board (No. IRB00001052-20025).

Funding: The National Key Research and Development Program, P.R. China (Grant No. 2020YFC0846300), National Natural Science Foundation of China (Grant No. 81673177; 41771527), and National Health Commission Capacity Building and Continuing Education Center (Grant No.: GWJJ2020100101).

\section{doi: $10.46234 / \mathrm{ccdcw} 2021.060$}

* Corresponding authors: Jiangli Di, dijiangli@chinawch.org.cn; Xiaohong Liu, 13522099566@163.com; Rongwei Ye, yerw@bjmu. edu.cn; Zhiwen Li, lizw@bjmu.edu.cn.

\footnotetext{
Institute of Reproductive and Child Health, Peking University/Key Laboratory of Reproductive Health, National Health Commission of the People's Republic of China, Beijing, China; ${ }^{2}$ Department of Epidemiology and Biostatistics, School of Public Health, Peking University, Beijing, China; ${ }^{3}$ National Center for Women and Children's Health, Chinese Center for Disease Control and Prevention,
}

Beijing, China; ${ }^{4}$ Department of Obstetrics and Gynecology, Haidian Maternal and Child Care Hospital, Beijing, China; ${ }^{5}$ College of Urban and Environmental Sciences, Peking University, Beijing, China; ${ }^{6}$ Institute of Geographic Sciences and Natural Resources Research, Chinese Academy of Sciences, Beijing, China; ${ }^{7}$ Key Laboratory of Geographic Information Science of the Ministry of Education, School of Geographic Sciences, East China Normal University, Shanghai, China.

\& Joint first authors.

Submitted: January 26, 2021; Accepted: February 22, 2021

\section{REFERENCES}

1. Goyal M, Singh P, Singh K, Shekhar S, Agrawal N, Misra S. The effect of the COVID-19 pandemic on maternal health due to delay in seeking health care: experience from a tertiary center. Int J Gynaecol Obstet 2021;52(2):231 - 5. http://dx.doi.org/10.1002/ijgo.13457.

2. Hu HQ, Huang AQ, Yang Q, Zhao W, Ma Y, Di JL, et al. Prevalence and risk factors of anemia of pregnant women -6 provinces in China, 2014-2018. China CDC Wkly 2020;2(14):225 - 9. http://dx.doi.org/ $10.46234 / \mathrm{ccdcw} 2020.058$.

3. Sentilhes L, De Marcillac F, Jouffrieau C, Kuhn P, Thuet V, Hansmann Y, et al. Coronavirus disease 2019 in pregnancy was associated with maternal morbidity and preterm birth. Am J Obstet Gynecol 2020;223(6):914.e1 - 15. http://dx.doi.org/10.1016/j.ajog. 2020.06.022.

4. Yan J, Guo JJ, Fan CF, Juan J, Yu XC, Li JF, et al. Coronavirus disease 2019 in pregnant women: a report based on 116 cases. Am J Obstet Gynecol 2020;223(1):111.e1 - 14. http://dx.doi.org/10.1016/j.ajog. 2020.04.014.

5. Khalil A, von Dadelszen P, Draycott T, Ugwumadu A, O'Brien P, Magee L. Change in the incidence of stillbirth and preterm delivery during the COVID-19 pandemic. JAMA 2020;324(7):705-6. http://dx.doi.org/10.1001/jama.2020.12746.

6. Justman N, Shahak G, Gutzeit O, Ben Zvi D, Ginsberg Y, Solt I, et al. Lockdown with a price: the impact of the COVID-19 pandemic on prenatal care and perinatal outcomes in a tertiary care center. Isr Med Assoc J 2020;22(9): 533-7. https://pubmed.ncbi.nlm.nih.gov/3323 6549/.

7. Li M, Yin H, Jin ZC, Zhang H, Leng BJ, Luo Y, et al. Impact of Wuhan lockdown on the indications of cesarean delivery and newborn weights during the epidemic period of COVID-19. PLoS One 2020;15(8):e0237420. http://dx.doi.org/10.1371/journal.pone.0237 420.

8. China State Council. Regulations about strengthing disease treatment and midwifery care for pregnant women during the COVID-19 pandemic in China. http://www.gov.cn/xinwen/2020-02/10/content 5476731.htm. and http://www.nhc.gov.cn/fys/s3581/202002/4f80657 b346e4d6ba76e2cfc3888c630.shtml. [2020-2-10]. (In Chinese).

9. Wu YT, Zhang C, Liu H, Duan CC, Li C, Fan JX, et al. Perinatal depressive and anxiety symptoms of pregnant women during the coronavirus disease 2019 outbreak in China. Am J Obstet Gynecol 2020;223(2):240.e1 - 9. http://dx.doi.org/10.1016/j.ajog.2020.05.009.

10. Jia XQ, Chen JX, Li LJ, Jia N, Jiangtulu B, Xue T, et al. Modeling the prevalence of asymptomatic COVID-19 infections in the Chinese mainland. The Innovation 2020;1(2):100026. http://dx.doi.org/10.1016/ j.xinn.2020.100026. 